\title{
Effect of coordinated probiotic/prebiotic/phytobiotic supplementation on microbiome balance and psychological mood state in healthy stressed adults
}

\author{
Shawn M. Talbott ${ }^{1}$, Julie A. Talbott ${ }^{2}$, Bret J. Stephens ${ }^{3}$, and Marc P. Oddou ${ }^{3}$ \\ ${ }^{1}$ Amare Global, Irvine, CA, USA; ${ }^{2}$ EQQIL, Draper, UT, USA; ${ }^{3}$ Wasatch Scientific, Murray, UT, \\ USA
}

Corresponding author: Shawn Talbott, PhD, Nutritional Biochemist, Amare Global, Irvine, CA, USA

Submission Date: February $8^{\text {th }}$, 2019, Acceptance Date: April $28^{\text {th }}$, 2019, Publication Date: April 31 ${ }^{\text {st }}, 2019$

Citation: Talbott SM., Talbott J.A., Stephens B.J., Oddou M.P. Effect of coordinated probiotic/prebiotic/phytobiotic supplementation on microbiome balance and psychological mood state in healthy stressed adults. Functional Foods in Health and Disease 2019; 9(4): 265-275. DOI: https://doi.org/10.31989/ffhd.v9i4.599

\begin{abstract}
Background: Interest in and knowledge of the gut microbiome has increased exponentially in the past decade. This once overlooked component of the gastrointestinal tract is now implicated in multiple aspects of human health, including mental (e.g. depression, anxiety, stress), metabolic (e.g. diabetes/obesity), neurological (e.g. Alzheimer's, Parkinson's, Autism Spectrum Disorder), gastrointestinal (e.g. irritable bowel syndrome, Crohn's), and immunological (e.g. inflammation, cancer) wellness, among others. Previous research has demonstrated the "strain specificity" of probiotic therapy (e.g. Lactobacillus helveticus R0052 for serotonin/depression; Bifidobacterium longum R0175 for GABA/anxiety; Lactobacillus rhamnosus R0011 for cortisol/stress). Similarly, probiotic bacteria demonstrate different growth trajectories based on availability of preferred fiber substrates (e.g. prebiotics) and phytonutrients such as flavonoids/polyphenols (e.g. phytobiotics). Thus, our objective was two-fold: to determine the change in microbiome ecology/balance and to evaluate the psychological mood state following a coordinated pro-/pre-/phyto-biotic supplementation regimen.
\end{abstract}

Methods: Thirty-two healthy subjects screened for "moderate" levels of psychological stress were randomly assigned to 1-month of Supplement (Amare Fundamentals, N=21) or matching Placebo $(\mathrm{N}=11)$. Microbiome balance was assessed in fecal samples using a PCR-based analysis (BiomeTracker) that has previously compared favorably to $16 \mathrm{~S}$ sequencing for abundance quantification for major phyla/families of bacteria. Psychological mood state parameters were assessed using the validated Profile of Mood States survey (POMS) to generate scores for Global Mood State, and six sub-scales (Depression, Tension, Fatigue, Anger, Confusion, and Vigor). 
Results: Following supplementation, there was a significant increase in populations of "good" bacteria in the Supplement group (+28\% Lactobacillus; $+30 \%$ Bifidobacterium) and overall composite score $(+17 \%)$ versus Placebo $(\mathrm{p}<0.05)$. Psychological indices were significantly improved in the Supplement group for both positive ( $+25 \%$ Global Mood; $+44 \%$ Vigor) and negative (-64\% Fatigue; -55\% Depression; -54\% Anger; -45\% Tension; $-43 \%$ Confusion) mood state parameters versus Placebo $(\mathrm{p}<0.05)$.

Conclusion: The World Health Organization has identified mental wellness issues as the leading contributor to global health burden - highlighting the urgency to develop lifestyle interventions to effectively manage depression, anxiety, and stress. These results demonstrate the close relationship between microbiome balance and psychological parameters - and the utility of targeted supplementation to positively influence the gut-brain-axis for improved mental wellness.

Keywords: Lactobacillus helveticus, Bifidobacterium longum, Lactobacillus rhamnosus, depression, anxiety, stress, vigor, mood state, mental wellness

\section{INTRODUCTION}

The gut-brain-axis describes the interconnected bidirectional communication between our "two brains" in the gut and the head $[1,2]$. Signaling pathways (the "axis") include nervous system (neural impulses and neurotransmitters), endocrine system (hormones), immune system (cytokines), and predominantly the 100 trillion intestinal bacteria that comprise the microbiome [ 3 -6]. The microbiome, in addition to its important roles in digestion, establishment/maintenance of the intestinal lining, and education/orchestration of immune regulation, is also known to produce a wide range of neuroactive compounds (serotonin, dopamine, norepinephrine, endocannabinoids/cannabinoid receptors, GABA, SCFAs, etc.) that reach the brain and peripheral tissues to regulate pain, immune vigilance, inflammation, mood, behavior, and gene regulation [2, 4]. The bi-directional nature of gut-brain communication is readily apparent from observations of classic gut-brain disorders such as irritable bowel syndrome (IBS), inflammatory bowel disease (IBD, Crohn's, ulcerative colitis), and autism - where psychological stress is known to disrupt microbiome balance (dysbiosis) and increase gastrointestinal permeability (leaky gut), while microbiome disturbances are known to influence emotional behavior [3-9].

Rodent studies have demonstrated mechanistically that modification of microbiome ecology can significantly and positively alter behavior (introversion/extraversion), metabolism (lean/obese), and mood (depression/anxiety) as well as potentially modify the trajectory of various disease states including certain cancers, Alzheimer's, Parkinson's, Chrohn's, autism, diabetes/obesity, and auto-immune diseases [1,2,4]. Similarly, recent human studies have shown modulation of microbiome structure/function to improve various indices of depression, anxiety, stress/resilience, chronic fatigue, and sleep quality [10-13]. For example, significant reductions in psychological distress were observed in subjects supplemented with probiotic bacteria (Lactobacillus helveticus R0052 and Bifidobacterium longum R0175), suggesting that naturally occurring probiotics may serve as preventive or therapeutic for depression [2, 4, 5, 14-17].

The World Health Organization has described mental wellness issues (e.g. depression, anxiety, stress, burnout) as the leading cause of "global disease burden" and as the "health epidemic of the $21^{\text {st }}$ century" [18-20], underscoring the urgent need for development of safe and effective interventions to alleviate global suffering. A range of natural functional food ingredients may fulfill this promise [5, 6], including probiotics (beneficial bacteria), prebiotics (fermentable 
fibers), and phytobiotics (phytonutrients with gut-health-promoting benefits). In particular, most [4-6, 10, 11], but not all [12] human trials have recently shown positive psychological outcomes for a number of nutrients including specific Lactobacillus (helveticus R0052; rhamnosus R0011) and Bifidobacterium (longum R0175) strains [5, 14-15]; unique oligosaccharide structures including galactooligosaccharide (GOS), isomaltooligosaccharide (IMO), and galactomannan [2133]; and traditional herbal extracts such as polyphenol/flavonoids [34]; ashwagandha [35]; magnolia bark [36]; rafuma [37]; sceletium [38]; amino acids [39-41]; and various phytonutrients [42-50].

Considering the magnitude of the problem (mental wellness issues as the leading cause of global disease burden), the inadequacy of existing treatments (poor efficacy/side effect ratio of prescription antidepressants), and the emerging evidence for positive psychological benefits of functional food remedies targeting the microbiome and gut-brain-axis [51-53], our objective was to determine the change in microbiome ecology/balance and psychological mood state following a coordinated pro-/pre-/phyto-biotic supplementation regimen.

\section{METHODS}

\section{Study design}

This study was done in accordance with the Helsinki Declaration, as revised in 1983, for clinical research involving humans, and all procedures, measurements, and informed consent processes were reviewed and approved by an external third-party review board (Aspire IRB; Santee, CA).

Subjects signed informed consent documents after the study details were explained. The study used a randomized, placebo-controlled, double-blind design. Subjects were randomly assigned, through a random number generator, to either Supplement (FundaMentals; Amare Global, Irvine, CA) or a matching Placebo (corn starch). Bottles were labeled with a pre-assigned blinded code. Subjects self-administered the allotted supplement/placebo once daily in the morning with breakfast for 4 weeks. Subjects were contacted weekly to remind them to take their supplement daily. Empty bottles were returned after the study for a count of any unused product (an indicator of missed doses). Compliance with these instructions was very high (data not shown).

We screened and randomly assigned 33 healthy subjects for "moderate" levels of psychological stress ("healthy stressed") and who were free of prescription antidepressants or antianxiety medications. One subject was lost to follow-up, resulting in 32 subjects completing the 1month Supplementation intervention (FundaMentals, $\mathrm{N}=21$; or matching Placebo, $\mathrm{N}=11$ ). The 1month duration was selected as more representative of persistent changes in mood state that may result from microbiome modulation and superior neurotransmitter balance, as opposed to shortterm changes in emotions that may be more closely linked with stressors of daily living.

\section{Dietary supplement}

Subjects in the Supplement group consumed one serving daily (in the morning, with or without breakfast) of a natural multi-ingredient targeted mental wellness supplement (FundaMentals, Amare Global, Irvine, CA, USA) containing probiotic bacteria, prebiotics fibers, and phytobiotic plant extracts and nutrients. In recognition of the recently demonstrated strain-specific benefits of probiotics and structure-specific benefits of prebiotics, the Supplement contained researchvalidated bacterial strains (e.g. Lactobaciluus helveticus R0052 for serotonin/depression; Bifidobacterium longum R0175 for GABA/anxiety; and Lactobacillus rhamnosus R0011 for cortisol/stress); clinically-proven prebiotic fibers (galactooligosaccharides, GOS, Bimuno, Clasado BioSciences, UK; and galactomannan, partially hydrolyzed guar gum, PHGG, Sunfiber, 
Taiyo International, Minneapolis MN USA); and selected nutrients with demonstrated mental wellness functional benefits across the gut-brain-axis, including L-theanine (Suntheanine, Taiyo International); Asian Apple Polyphenols (Applephenon, BGG, Beijing, China); French Grape Seed Polyphenols (Enovita, Indena, Milan, Italy); New Zealand Pine Bark Polyphenols (Enzogenol, Enzo, Auckland, New Zealand); and Artichoke Leaf Extract plus Ginger Root Extract (ProDigest, Indena, Milan Italy).

\section{Microbiome Assessment}

Microbiome analysis of fecal samples was carried out using the complete BiomeTracker system (Wasatch Scientific, Murray, UT). Briefly, Fecal samples were obtained by nylon swab and placed into preservative binding buffer to lock the composition of bacteria in place. DNA was then purified by following the recommended procedure and using the provided DNA columns. Reaction mixtures were set up as recommended with the components provided (WS\#1- WS\#8), and 20ng of DNA from each sample was added to the reaction mixtures. Samples were processed using the recommended conditions on an ABI 7500 Fast (Applied Biosystems) instrument in duplicate. Threshold values were input into the normalization/quantification template provided.

\section{Mood Assessment}

We employed the Profile of Mood States (POMS) questionnaire [54-55] to measure 6 primary psychological factors (tension, depression, anger, fatigue, vigor, or confusion) plus the combined global mood state as an indication of subjective well-being. The POMS methodology has been used in $~ 3,000$ studies [55], and its validity is well established [54]. The POMS profile uses 65 adjective-based intensity scales scored on a 0-4 hedonic scale (e.g. "not at all" to "extremely"). The 65 adjective responses are categorized into the 6 mood factors (tension, depression, anger, fatigue, vigor, or confusion), tabulated, scored, and analyzed. The output of the POMS questionnaire is an assessment of the positive and negative moods of each subject at baseline and post-supplementation (4 weeks).

\section{Data Management and Analysis}

All questionnaires were mailed to a central location and transcribed to a central database. Subjects who did not complete the questionnaires or who submitted incomplete questionnaires were dropped from the study and not included in the study analysis. Data were identified by subject number and examined for accuracy and completeness. Tabulated data were analyzed with JMP 14.0 (SAS Institute, Cary, NC) using standard parametric paired t tests, and significance was assessed with a 2-tailed alpha level set at 0.05 .

\section{RESULTS AND DISCUSSION}

Following supplementation, there was a significant increase in populations of "good" bacteria in the Supplement group (+28\% Lactobacillus; $+30 \%$ Bifidobacterium) and overall composite score $(+17 \%)$ versus Placebo $(\mathrm{p}<0.05)$. Psychological indices were significantly improved in the Supplement group for both positive ( $+25 \%$ Global Mood - lower score indicates superior wellbeing, Figure 2A; and $+44 \%$ Vigor, Figure 2B) and negative (-64\% Fatigue; $-55 \%$ Depression; 54\% Anger; $-45 \%$ Tension; -43\% Confusion, Figure 2B) mood state parameters versus Placebo $(\mathrm{p}<0.05)$. 
A

Lactobacillus

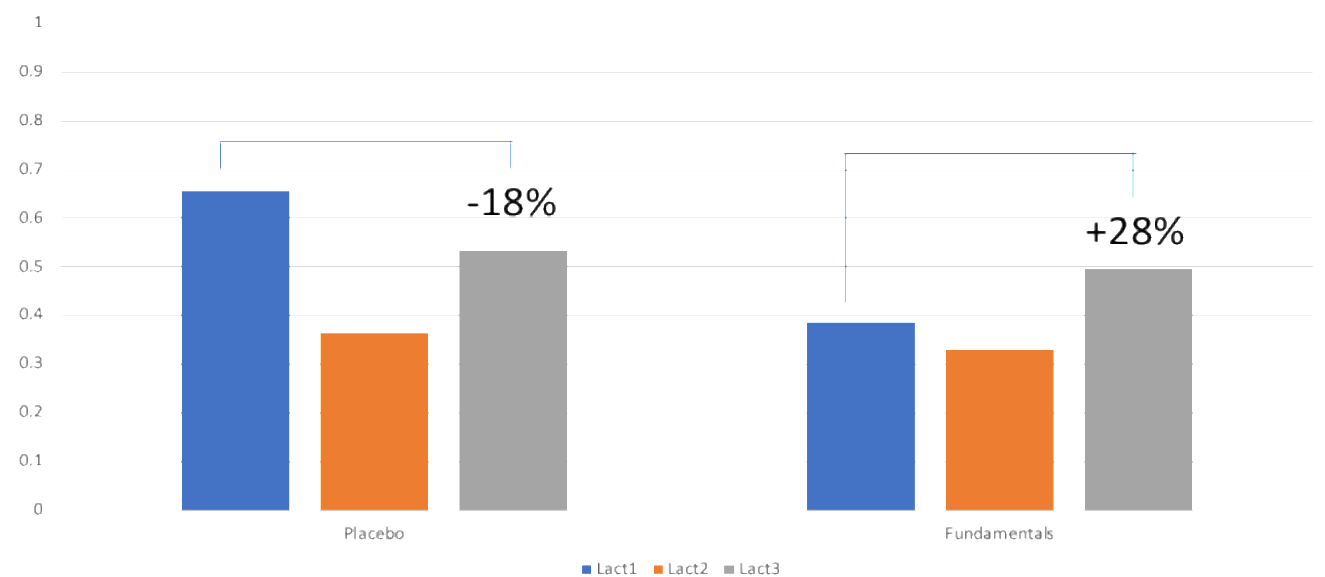

B

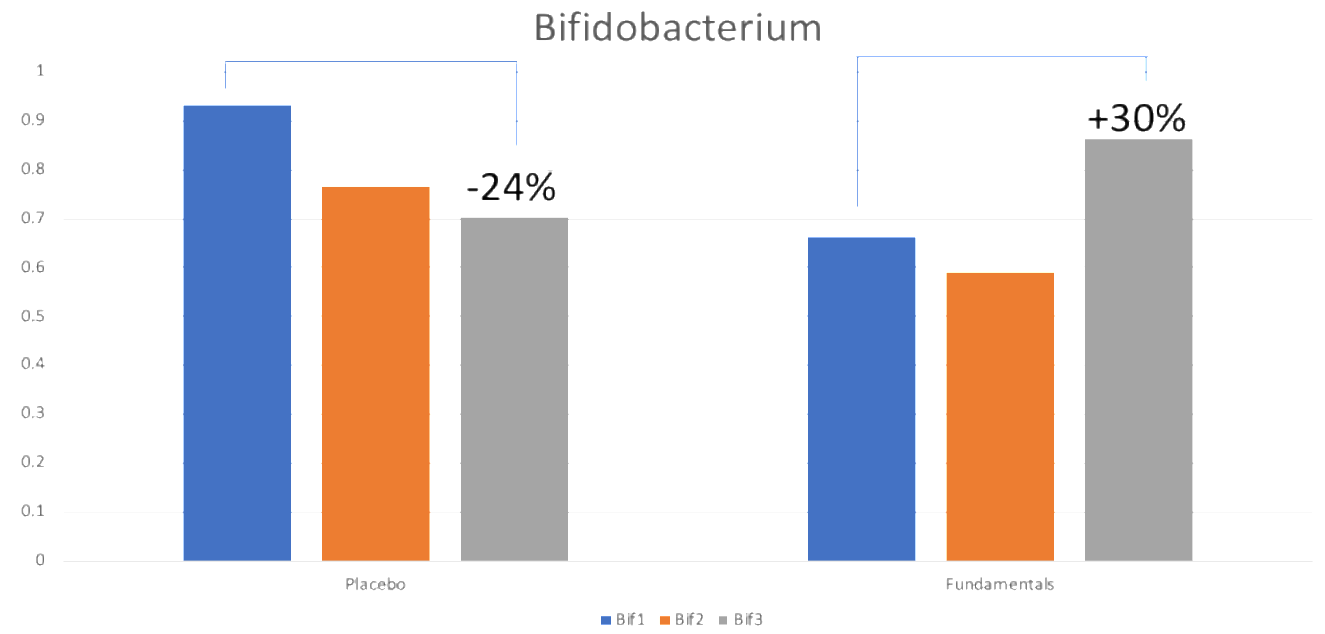

C

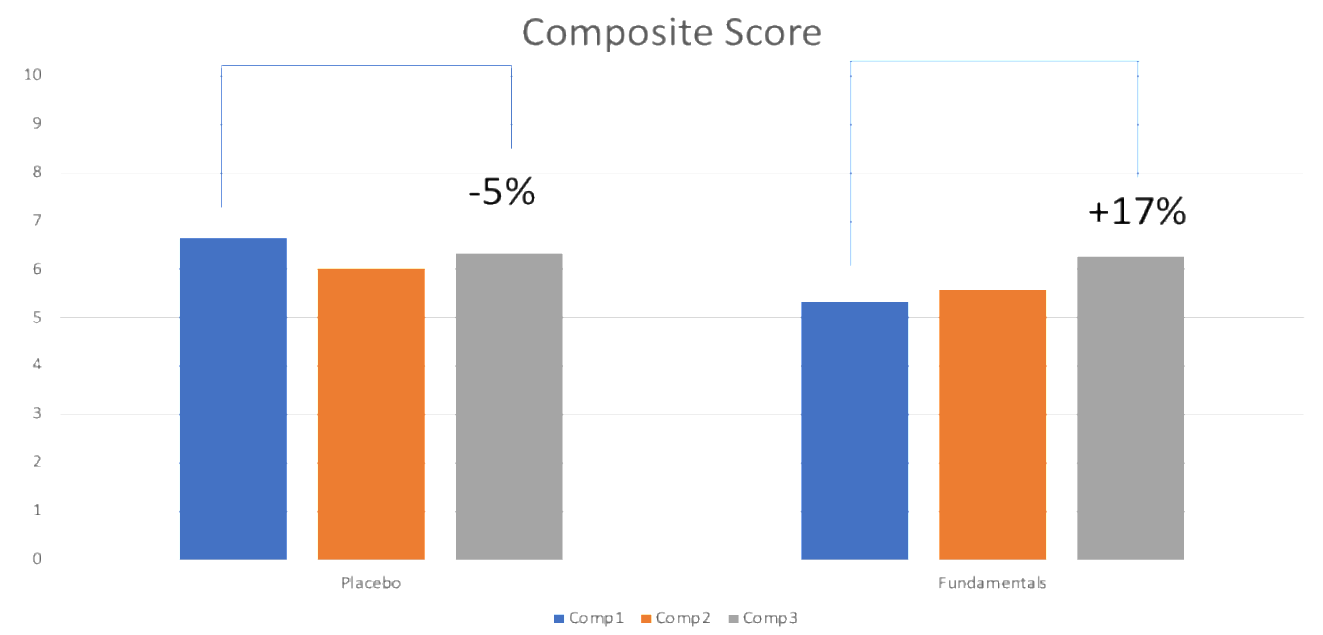

Figure 1 A-C. Microbiome Results

Following supplementation, there was a significant increase in populations of "good" bacteria in the Supplement group (+28\% Lactobacillus, Figure 1A; $+30 \%$ Bifidobacterium, Figure 1B) and overall composite score $(+17 \%$, Figure $1 \mathrm{C})$ versus Placebo $(\mathrm{p}<0.05)$. 
A

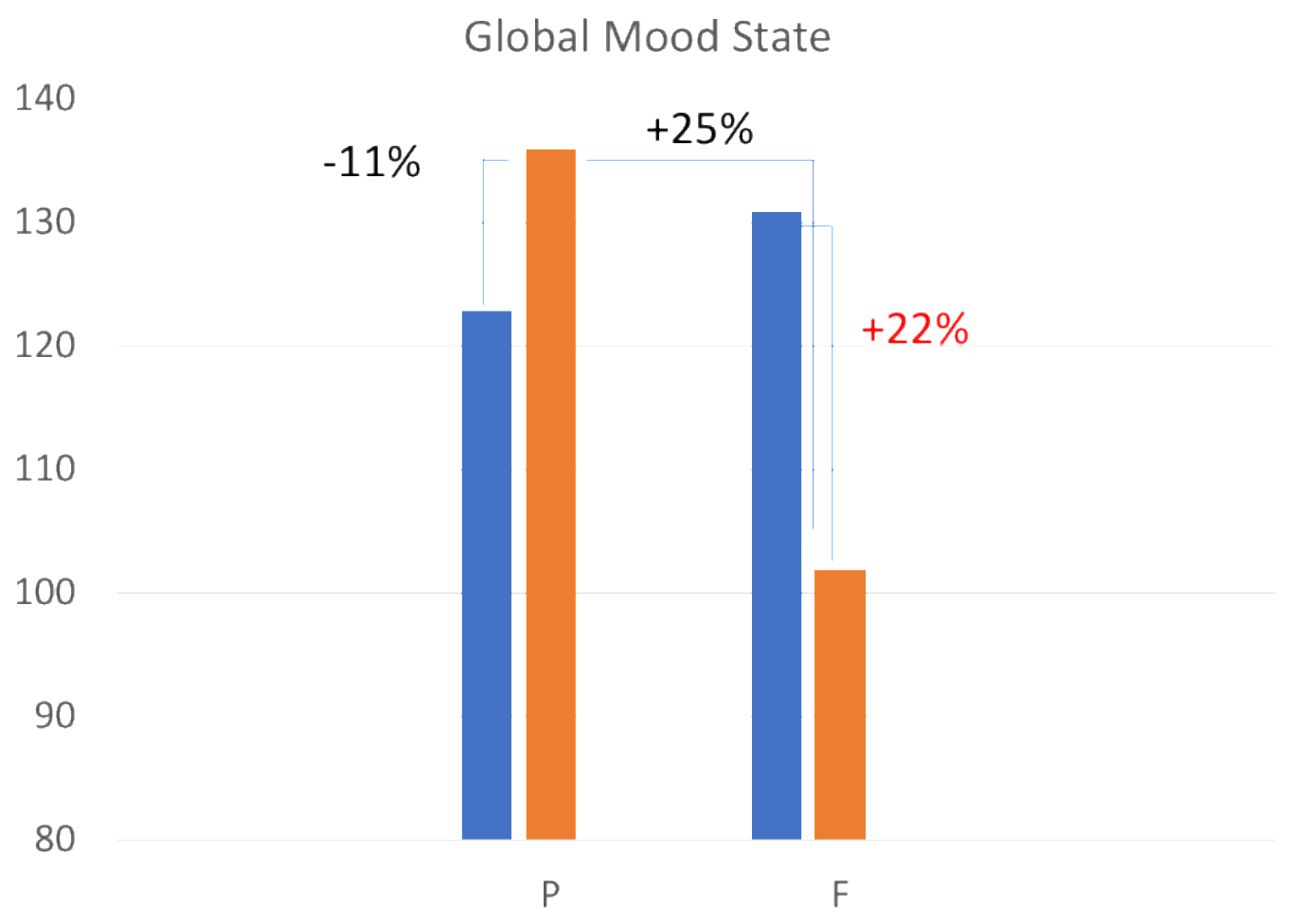

B

\section{Mood State Sub-Scales}

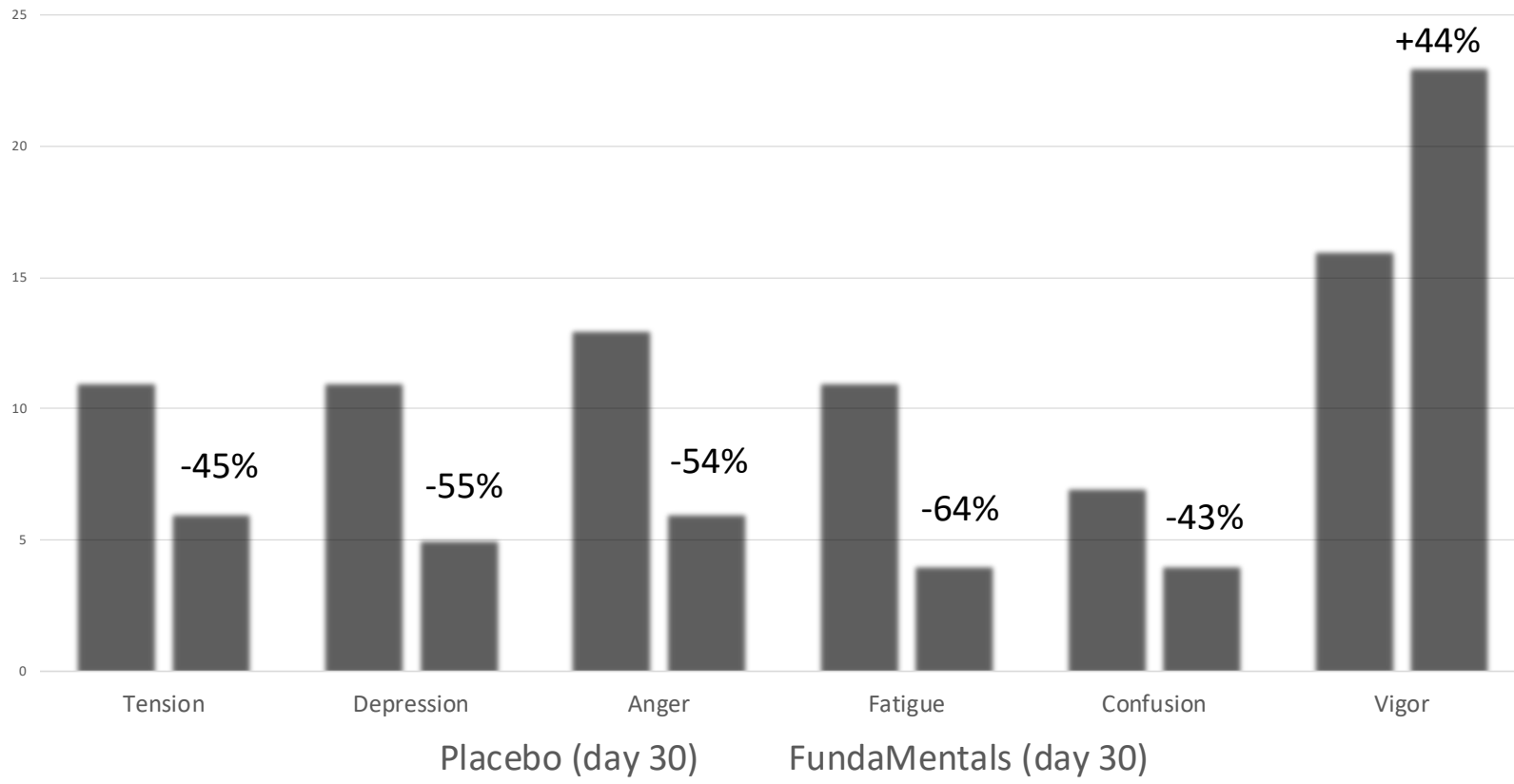

Figure 2 A-B. Psychological Mood State Results

Psychological indices were significantly improved in the Supplement group for both positive (+25\% Global Mood, Figure 2A; +44\% Vigor, Figure 2B) and negative (-45\% Tension; $-55 \%$ Depression; -54\% Anger; -64\% Fatigue; -43\% Confusion, Figure 2C) mood state parameters versus Placebo $(\mathrm{p}<0.05)$. 
Previous studies have demonstrated psychological benefits of probiotic, prebiotic, and phytobiotic formulations in healthy human volunteers, including lowered stress, anxiety and depression [14-15, 23, 39-42]. For example, dietary polyphenols/flavonoids, which have welldescribed benefits for cardiovascular, neurologic, and metabolic health are increasingly appreciated to also be involved in supporting gastrointestinal function and integrity by modulating microbiome balance (increase of "good" and decrease of "bad" bacteria species), which further supports mental wellness benefits through increased production and efficiency of beneficial signaling molecules [49-50]. The observed mental wellness benefits may be related to improvements in microbiome ecology [26-27, 31, 43-44, 48-50], gut integrity [29, 30, 45, 47, 48] and more efficient signaling across multiple interconnected biochemical pathways including reduced inflammation and cortisol exposure [14-16, 22, 25, 27], improved serotonin/GABA/SCFA signaling [16-17, 26-27, 32-33], enhanced immune function [27], and without noticeable or reported side effects [7, 10, 12, 14-15, 22-23, 27-28].

The last decade has seen our understanding of the interconnected and interdependent nature of the microbiome-gut-brain-axis and its role in modulating the entire mental wellness continuum from depression/anxiety to fatigue/stress to optimal mental and physical performance (e.g. vigor). Such understanding provides tantalizing potential for therapeutic interventions - as well as prevention strategies - for some of our most persistent and resistant public health challenges, including anxiety, depression, attention-deficit hypersensitivity disorder (ADHD), autism, posttraumatic stress disorder (PTSD), chronic fatigue syndrome, fibromyalgia, and many others related to chronic stress, inflammation, and immune system disruption.

\section{CONCLUSION}

The World Health Organization has identified mental wellness issues as the leading contributor to global health burden - highlighting the urgency to develop lifestyle interventions to effectively manage depression, anxiety, and stress. These results demonstrate the close relationship between microbiome balance and psychological parameters - and the utility of targeted supplementation to positively influence the gut-brain-axis for improved mental wellness.

List of Abbreviations: GABA, gamma-Aminobutyric acid; PCR, Polymerase chain reaction; POMS, Profile of Mood States survey; SCFAs, Short-chain fatty acids; GOS, galactooligosaccharide; IMO isomaltooligosccharide.

Competing Interests: S.M.T. is an employee of Amare Global, the producer of the FundaMentals dietary supplement.

Authors' Contributions: S.M.T. designed the research protocol. J.T. coordinated the IRB submission, subject recruitment, and study monitoring. B.J.S. and M.J.O. performed and oversaw the microbiome assessments. All authors were involved in the preparation and presentation of these data.

Acknowledgements and Funding: The authors would like to thank the volunteers who contributed their samples, time, and focus to this study, including Amare Global for providing the FundaMentals dietary supplements and funding the costs associated with microbiome assessments and psychological surveys. 


\section{REFERENCES}

1. Mayer, EA: Gut feelings: the emerging biology of gut-brain communication. Nat Rev Neurosci 2011, 12 (8): 453-466.

2. Mayer EA, Knight R, Mazmanian SK, Cryan JP, Tillisch K: Gut microbes and the brain: paradigm shift in neuroscience. J Neurosci 2014, 34 (46): 15490-15496.

3. Mayer EA, Naliboff BD, Craig AD: Neuroimaging of the brain-gut axis: from basic understanding to treatment of functional GI disorders. Gastroenterology 2006, 131 (6): 1925-1942.

4. Evrensel A, Ceylan ME: The Gut-Brain Axis: The Missing Link in Depression. Clin Psychopharmacol Neurosci 2015, 13(3): 239-44.

5. Wallace CJK, Milev R: The effects of probiotics on depressive symptoms in humans: a systematic review. Ann Gen Psychiatry 2017, 16:14.

6. McKean J, Naug H, Nikbakht E, Amiet B, Colson N: Probiotics and Subclinical Psychological Symptoms in Healthy Participants: A Systematic Review and Meta-Analysis. J Altern Complement Med 2017, 23(4):249-258.

7. Rousseaux C, Thuru X, Gelot A, et al.: Lactobacillus acidophilus modulates intestinal pain and induces opioid and cannabinoid receptors. Nat Med 2007, 3:35-37.

8. Cani PD, Plovier H, Van Hul M, Geurts L, Delzenne NM, Druart C, Everard A: Endocannabinoids--at the crossroads between the gut microbiota and host metabolism. Nat Rev Endocrinol 2016, 12(3):133-43.

9. DiPatrizio, Nicholas V: Endocannabinoids in the Gut. Cannabis and Cannabinoid Research 2016, 1(1): 67-77.

10. Steenbergen L, Sellaro R, van Hemert S, Bosch JA, Colzato LS: A randomized controlled trial to test the effect of multispecies probiotics on cognitive reactivity to sad mood. Brain Behav Immun 2015, 48: 258-64.

11. Romijn AR, Rucklidge JJ: Systematic review of evidence to support the theory of psychobiotics. Nutr Rev 2015, 73(10): 675-93.

12. Romijn AR, Rucklidge JJ, Kuijer RG, Frampton C: A double-blind, randomized, placebocontrolled trial of Lactobacillus helveticus and Bifidobacterium longum for the symptoms of depression. Aust N Z J Psychiatry 2017, 51(8): 810-821.

13. Nakagawa M, Yamamoto H, Kawaji M, Miura N, Wakame K, Endo T: Effects of lactic acid bacteria-containing foods on the quality of sleep: a placebo-controlled, double- blinded, randomized crossover study. Functional Foods in Health and Disease 2018, 8(12): 579-596.

14. Messaoudi M, Lalonde R, Violle N, Javelot H, Desor D, Nejdi A, et al.: Assessment of psychotropic-like properties of a probiotic formulation (Lactobacillus helveticus R0052 and Bifidobacterium longum R0175) in rats and human subjects. Br J Nutr 2011, 105(5):755-64.

15. Messaoudi M, Violle N, Bisson JF, Desor D, Javelot H, Rougeot C: Beneficial psychological effects of a probiotic formulation (Lactobacillus helveticus R0052 and Bifidobacterium longum R0175) in healthy human volunteers. Gut Microbes 2011, 2(4): 256-261.

16. Savignac HM, Tramullas M, Kiely B, Dinan TG, Cryan JF: Bifidobacteria modulate cognitive processes in an anxious mouse strain. Behav Brain Res 2015, 287: 59-72. 
17. Bravo JA, Forsythe P, Chew MV, Escaravage E, Savignac HM, Dinan TG, et al.: Ingestion of Lactobacillus strain regulates emotional behavior and central GABA receptor expression in a mouse via the vagus nerve. Proc Natl Acad Sci U S A. 2011, 108(38): 16050-5.

18. Becker AE, Kleinman A: Mental health and global agenda. NEJM 2013, 369: 66-73.

19. Ferrari AJ, Charlson FJ, Norman RE, Patten SB, Freedman G, Murray CJ, et al.: Burden of depressive disorders by country, sex, age, and year: findings from the global burden of disease study 2010. PLoS Med 2013, 10(11): e1001547.

20. World Health Organization. Mental health atlas [http://apps.who.int/iris/bitstream/ 10665/44697/1/9799241564359_eng.pdf]

21. Savignac HM, Couch Y, Stratford M, Bannerman DM, Tzortzis G, Anthony DC, et al.: Prebiotic administration normalizes lipopolysaccharide (LPS)-induced anxiety and cortical 5-HT2A receptor and IL1- $\beta$ levels in male mice. Brain Behav Immun 2016, 52: 120-31.

22. Vulevic J, Juric A, Walton GE, Claus SP, Tzortzis G, Toward RE, et al.: Influence of galactooligosaccharide mixture (B-GOS) on gut microbiota, immune parameters and metabonomics in elderly persons. Br J Nutr 2015, 114(4): 586-95.

23. Schmidt K, Cowen PJ, Harmer CJ, Tzortzis G, Errington S, Burnet PW: Prebiotic intake reduces the waking cortisol response and alters emotional bias in healthy volunteers. Psychopharmacology (Berl) 2015, 232(10): 1793-801.

24. Grimaldi R, Cela D, Swann JR, Vulevic J, Gibson GR, Tzortzis G, et al.: In vitro fermentation of B-GOS: impact on faecal bacterial populations and metabolic activity in autistic and non-autistic children. FEMS Microbiol Ecol 2017, 93(2).

25. Liu Y, Gibson GR, Walton GE: An In Vitro Approach to Study Effects of Prebiotics and Probiotics on the Faecal Microbiota and Selected Immune Parameters Relevant to the Elderly. PLoS One 2016, 11(9): e0162604.

26. Grimaldi R, Swann JR, Vulevic J, Gibson GR, Costabile A: Fermentation properties and potential prebiotic activity of Bimuno ${ }^{\circledR}$ galacto-oligosaccharide $(65 \%$ galactooligosaccharide content) on in vitro gut microbiota parameters. Br J Nutr 2016, 116(3): 4806.

27. Vulevic J, Juric A, Tzortzis G, Gibson GR: A mixture of trans-galactooligosaccharides reduces markers of metabolic syndrome and modulates the fecal microbiota and immune function of overweight adults. J Nutr 2013, 143(3): 324-31.

28. Vulevic J, Drakoularakou A, Yaqoob P, Tzortzis G, Gibson GR: Modulation of the fecal microflora profile and immune function by a novel trans-galactooligosaccharide mixture (BGOS) in healthy elderly volunteers. Am J Clin Nutr 2008, 88(5):1438-46.

29. Quartarone G: Role of PHGG as a dietary fiber: a review article. Minerva Gastroenterol Dietol 2013, 59(4):329-40.

30. Giannini EG, Mansi C, Dulbecco P, Savarino V: Role of partially hydrolyzed guar gum in the treatment of irritable bowel syndrome. Nutrition 2006, 22(3): 334-42.

31. Carlson J, Gould T, Slavin J: In vitro analysis of partially hydrolyzed guar gum fermentation on identified gut microbiota. Anaerobe 2016, 42: 60-66. 
32. Takagi T, Naito Y, Higashimura Y, Ushiroda C, Mizushima K, Ohashi Y, et al.: Partially hydrolysed guar gum ameliorates murine intestinal inflammation in association with modulating luminal microbiota and SCFA. Br J Nutr 2016, 116(7): 1199-1205.

33. Ohashi Y, Sumitani K, Tokunaga M, Ishihara N, Okubo T, Fujisawa T: Consumption of partially hydrolysed guar gum stimulates Bifidobacteria and butyrate-producing bacteria in the human large intestine. Benef Microbes 2015, 6(4): 451-5.

34. Gutierrez-Merino C, Lopez-Sanchez C, Lagoa R, Samhan-Arias AK, Bueno C, GarciaMartinez V: Neuroprotective actions of flavonoids. Curr Med Chem 2011, 18(8): 1195-212.

35. Pratte MA, Nanavati KB, Young V, Morley CP: An alternative treatment for anxiety: a systematic review of human trial results reported for the Ayurvedic herb ashwagandha (Withania somnifera). J Altern Complement Med 2014, 20(12): 901-8.

36. Kalman DS, Feldman S, Feldman R, Schwartz HI, Krieger DR, Garrison R: Effect of a proprietary Magnolia and Phellodendron extract on stress levels in healthy women: a pilot, double-blind, placebo-controlled clinical trial. Nutr J 2008, 7:11.

37. Xie W, Zhang X, Wang T, Hu J: Botany, traditional uses, phytochemistry and pharmacology of Apocynum venetum L. (Luobuma): A review. J Ethnopharmacol 2012, 141(1): 1-8.

38. Nell H, Siebert M, Chellan P, Gericke N: A randomized, double-blind, parallel-group, placebo-controlled trial of Extract Sceletium tortuosum (Zembrin) in healthy adults. J Altern Complement Med 2013, 19(11): 898-904.

39. Unno K, Tanida N, Ishii N, Yamamoto H, Iguchi K, Hoshino M, et al.: Anti-stress effect of theanine on students during pharmacy practice: positive correlation among salivary $\alpha$ amylase activity, trait anxiety and subjective stress. Pharmacol Biochem Behav 2013, 111:128-35.

40. White DJ, de Klerk S, Woods W, Gondalia S, Noonan C, Scholey AB: Anti-Stress, Behavioural and Magnetoencephalography Effects of an L-Theanine-Based Nutrient Drink: A Randomised, Double-Blind, Placebo-Controlled, Crossover Trial. Nutrients 2016, 8(1).

41. Kimura K, Ozeki M, Juneja LR, Ohira H: L-Theanine reduces psychological and physiological stress responses. Biol Psychol 2007, 74(1): 39-45.

42. Ataka S, Tanaka M, Nozaki S, Mizuma H, Mizuno K, Tahara Tet al.: Effects of Applephenon and ascorbic acid on physical fatigue. Nutrition 2007, 23(5): 419-23.

43. Koutsos A, Tuohy KM, Lovegrove JA: Apples and cardiovascular health--is the gut microbiota a core consideration? Nutrients 2015, 7(6): 3959-98.

44. Espley RV, Butts CA, Laing WA, Martell S, Smith H, McGhie TK, et al.: Dietary flavonoids from modified apple reduce inflammation markers and modulate gut microbiota in mice. $\mathrm{J}$ Nutr 2014, 144(2): 146-54.

45. Graziani G, D'Argenio G, Tuccillo C, Loguercio C, Ritieni A, Morisco F, et al.: Apple polyphenol extracts prevent damage to human gastric epithelial cells in vitro and to rat gastric mucosa in vivo. Gut 2005, 54(2): 193-200.

46. Liu W, Zhao S, Wang J, Shi J, Sun Y, Wang W, et al.: Grape seed proanthocyanidin extract ameliorates inflammation and adiposity by modulating gut microbiota in high-fat diet mice. Mol Nutr Food Res, 2017. 
47. Gil-Cardoso K, Ginés I, Pinent M, Ardévol A, Arola L, Blay M, et al.: Chronic supplementation with dietary proanthocyanidins protects from diet-induced intestinal alterations in obese rats. Mol Nutr Food Res, 2017.

48. Zhou L, Wang W, Huang J, Ding Y, Pan Z, Zhao Y, et al.: In vitro extraction and fermentation of polyphenols from grape seeds (Vitis vinifera) by human intestinal microbiota. Food Funct 2016, 7(4): 1959-67.

49. Anhê FF, Varin TV, Le Barz M, Desjardins Y, Levy E, Roy D, et al.: Gut Microbiota Dysbiosis in Obesity-Linked Metabolic Diseases and Prebiotic Potential of Polyphenol-Rich Extracts. Curr Obes Rep 2015, 4(4): 389-400.

50. Klinder A, Shen Q, Heppel S, Lovegrove JA, Rowland I, Tuohy KM: Impact of increasing fruit and vegetables and flavonoid intake on the human gut microbiota. Food Funct 2016, 7(4): 1788-96.

51. Marchesi JR, Adams DH, Fava F, Hermes GD, Hirschfield GM, Hold G, et al.: The gut microbiota and host health: a new clinical frontier. Gut 2016, 65(2): 330-9.

52. Petra AI, Panagiotidou S, Hatziagelaki E, Stewart JM, Conti P, Theoharides TC: GutMicrobiota-Brain Axis and Its Effect on Neuropsychiatric Disorders With Suspected Immune Dysregulation. Clin Ther 2015, 37(5): 984-95.

53. Caracciolo B, Xu W, Collins S, Fratiglioni L: Cognitive decline, dietary factors and gutbrain interactions. Mech Ageing Dev 2014, 136-137: 59-69.

54. McNair DM, Lorr M, Droppleman LF: Manual for the Profile of Mood States. San Diego, CA: Educational and Industrial Testing Services; 1971.

55. Leunes A: Updated bibliography on the profile of mood states in sport and exercise psychology research. J Appl Sport Psychol 2000, 12:110-113. 